\title{
Trente jours en Sierra Leone
}

\author{
Brooks, $\mathrm{JI}^{1,2^{*}}$ \\ ${ }^{1}$ Laboratoire national de microbiologie, Agence de la santé publique du Canada, Winnipeg (Manitoba) \\ ${ }^{2}$ Centre de l'immunisation et des maladies respiratoires infectieuses, Agence de la santé publique du Canada, Ottawa \\ (Ontario)
}

*Correspondance : James.Brooks@phac-aspc.gc.ca

C'était le 29 septembre 2014. Ce jour-là, 6500 cas d'infection au virus Ebola ayant causé 3500 décès avaient été signalés en Afrique de l'Ouest, et le premier patient infecté par le virus Ebola aux États-Unis avait été admis à I'hôpital et était sur le point de transmettre le virus à deux infirmières. Le virus Ebola balayait l'Afrique de l'Ouest et frappait à la porte de l'Amérique du Nord. Je dirigeais l'un des laboratoires nationaux du VIH de l'Agence de la santé publique du Canada (ASPC) et le patron est entré dans mon bureau et a dit : « Brooks, si vous souhaitez apporter votre contribution à la santé publique, c'est le moment. »Quinze minutes plus tard, par téléconférence, je me portais volontaire pour effectuer les diagnostics du virus Ebola dans l'un des laboratoires mobiles de campagne des laboratoires nationaux de microbiologie (LNM) de l'ASPC en Sierra Leone.

En tant que médecin spécialiste des maladies infectieuses, je me suis toujours intéressé aux infections émergentes et cette éclosion de la maladie à virus Ebola représentait pour moi une occasion à saisir. Mon épouse et ma famille connaissaient ce vif intérêt et m'ont incité à partir. En quelques jours, j'avais reçu le feu vert de mon directeur, réorganisé mes consultations, organisé une couverture pour mes patients, transmis mes dossiers et rejoint Winnipeg en avion pour une formation en laboratoire. Les membres du personnel du LNM ont trouvé du temps dans leurs horaires chargés pour me fournir la formation garantissant que j'aurais les compétences requises. Le Centre des opérations du LNM m'a complètement équipé en me fournissant tout ce dont j'aurais besoin pour aller dans la jungle. Le personnel de la santé au travail m'a examiné pour me fournir un appareil de protection respiratoire et le personnel médical m'a fourni une trousse de premiers soins, une moustiquaire, de l'antimoustique et tout un tas d'antibiotiques. J'avais l'impression d'être dans la peau de James Bond et de recevoir une mission; ces gens-là savaient ce qu'ils faisaient. J'ai ensuite été renvoyé à Ottawa afin d'être examiné sur le plan médical pour pouvoir être déployé et j'ai reçu un certain nombre de vaccinations.

En raison du caractère fluctuant de l'épidémie ainsi que des exigences et de la disponibilité changeantes en matière de personnel, le déploiement suivait un rythme " On se dépêche et on attend », comme dans l'armée. À mesure que l'incertitude et l'angoisse montaient, j'ai commencé à penser que je ne serais peut-être jamais déployé. J'ai donc fait savoir à certaines personnes qu'il était probable que je parte, mais je ne voulais pas le dire à tout le monde au cas où ce ne serait finalement pas le cas. Toutefois, la nouvelle s'est répandue et j'ai été touché par le nombre de mes amis et collègues qui sont venus me voir simplement pour me remercier pour l'aide que j'allais apporter ou pour me dire qu'ils étaient fiers de moi. D'autres personnes ont proposé de conduire mes enfants aux entraînements de hockey ou de les emmener aux tournois. J'ai reçu des courriels d'amis qui savaient que mon épouse n'oserait pas demander de l'aide, mais m'ont supplié de m'adresser à eux s'il y avait besoin. Enfin, le 15 décembre, le voile de l'incertitude a été levé. Trois collègues et moi-même avons reçu un message indiquant que nous partions pour l'Afrique de l'Ouest le 30 décembre. Le groupe responsable des déplacements au LNM avait déjà nos billets. Tout ce que nous avions à faire, c'était de nous présenter à l'aéroport.

Le 31 décembre 2014, après un voyage de 36 heures, je me suis retrouvé sur le tarmac, dans la chaleur moite de l'aéroport international Lungi, à Freetown, en Sierra Leone. L'avion était plein d'expatriés provenant de différentes organisations non gouvernementales, tous là pour apporter leur aide. Lorsque je suis entré dans l'aérogare, j'ai reçu mon baptême Ebola en me lavant les mains à l'eau de javel, un rituel que j'allais répéter des milliers de fois 
au cours des trente jours suivants. En regardant autour de moi, j'ai vu que tout le monde avait l'air en sueur. Étaitce la chaleur ou ces personnes étaient-elles infectées par le virus Ebola? Je me suis posé la question. Une personne m'a effleuré. Whoa! J'ai fait un bond en arrière. La Sierra Leone est une zone où il est formellement interdit de se toucher. Et si cette personne était infectée par le virus Ebola? J'ai pensé : « Oh non, j'ai seulement passé cinq minutes ici et je suis infecté par le virus Ebola. » J'ai sorti mon petit flacon de Purell. C'était bon d'en mettre sur mes mains, c'était comme un bouclier apaisant et invisible. Ensuite, j'ai commencé à répéter mon mantra : «Remettre le passeport, Purell, prendre mes bagages, Purell, compter les millions de léones du change de devises, Purell, Purell, Purell. »

En sortant dans la nuit brumeuse, j'ai vu et senti la fumée de feux perpétuels qui provenaient de la brousse et qui nettoyaient et brûlaient les déchets. J'ai ensuite été immédiatement entouré d'hommes qui voulaient m'aider à porter mes bagages. J'ai traversé une aire de stationnement non revêtue et j'ai recherché désespérément dans l'obscurité un membre de Médecins Sans Frontières, l'organisation qui devait m'accueillir pendant les trente jours suivants. Les hommes qui m'avaient proposé de porter mes bagages voulaient ensuite de l'argent, avec deux de leurs amis qui sont apparus dans l'obscurité. "Sont-ils infectés par le virus Ebola? » Je me suis posé la question. J'ai sorti mon flacon de Purell. Au milieu du chaos tourbillonnant de chaleur, de fumée, de sueur et de cris qui s'en est suivi, le chauffeur de Médecins Sans Frontières est apparu et a pris mes bagages qui ont immédiatement disparu. Un reçu m'a été mis dans la main et m'a miraculeusement permis de retrouver mon sac à Freetown. Purell. En sortant de l'aire de stationnement, dans un minibus qui m'emmenait au traversier, le visage de l'un des hommes qui m'avait « aidé » à porter mon sac est apparu contre la vitre puis s'est évanoui dans la nuit. Bienvenue à Freetown.

Avant de rejoindre un hôtel très moderne qui devait servir de liaison pour mon voyage aller et retour, je devais prendre le traversier pour traverser le fleuve Sierra Leone jusqu'à Freetown. Le minibus a roulé jusqu'au terminal du traversier sur une route que je pensais naïvement être la route la plus cahoteuse possible. Une personne a appelé mon numéro de billet et j'ai marché sur un quai de 100 mètres dans une nuit d'encre où la rivière et le ciel ne faisaient plus qu'un. Un bateau ouvert avec des sièges pour vingt personnes est apparu dans l'obscurité. On m'a remis un gilet de sauvetage avec une fermeture à glissière cassée qui avait la texture et probablement la flottabilité d'une veste légère. Deux moteurs hors-bord Mercury de 250 chevaux se sont mis en marche et nous ont projetés dans le néant. De loin, la seule chose que je pouvais voir, c'était un énorme feu de forêt brûlant sur la rive éloignée. La traversée a été une expérience charnière pour moi. Les choses étaient totalement hors de mon contrôle, de façon telle que je n'abandonnerais jamais autant le contrôle en temps normal. À ce moment-là, je me suis rendu compte que, pour continuer, pour être capable de faire mon travail et d'être heureux, je devais m'abandonner à la Sierra Leone. Cette décision a fait toute la différence.

À $6 \mathrm{~h}$ le lendemain, mes trois collègues, moi-même, et tout l'équipement nécessaire pour réapprovisionner deux laboratoires, ont été chargés dans un Toyota Land Cruiser. On ne peut pas dire que c'était un safari cinq étoiles en Afrique. Ces Land Cruiser sont fabriqués d'après les spécifications de Médecins Sans Frontières. Cela signifie qu'il n'y a pas d'électronique, pas d'ABS, pas de dégivrage arrière, pas de climatisation, les pneus ont des chambres à air, on s'assoit sur des bancs durs sur les côtés et il n'y a pas de turbo sur ces véhicules diesel. Mais nous avions de la chance, notre chauffeur avait un chapeau de cowboy et nous nous sommes rapidement retrouvés à rouler dans la jungle en écoutant de la musique country... de la véritable musique country émouvante. Pendant les neuf heures suivantes, nous avons parcouru $300 \mathrm{~km}$ de l'ouest à l'est du pays sur des routes qui sont passées de routes revêtues, à des routes gravillonnées, à des routes en terre battue, puis à des routes qui ne ressemblaient pas à des routes. Pendant ce voyage épique, nos douloureux fessiers et tout notre matériel ont été transférés dans cinq véhicules consécutifs jusqu'à ce que notre dernier véhicule, Bravo $(B)-4$, roule jusqu'à « Kilo Base ", le complexe hôtelier Luawa de Kailahun. En sortant dans la chaleur torride à 35 degrés, quelqu'un a pris ma température, je me suis de nouveau lavé les mains dans de l'eau de javel, j'ai présenté les semelles de mes 
chaussures pour qu'elles soient vaporisées avec de l'eau de javel avant d'entrer dans ce qui serait ma maison pour les trente jours suivants.

J'ai avancé péniblement dans la chaleur, saisi une boisson et me suis dirigé vers le coin-repas pour rencontrer quelques-uns de mes nouveaux collègues de Médecins Sans Frontières. Non seulement les membres de Médecins Sans Frontières étaient courageux et avaient effectué des missions en Somalie ou au Soudan du Sud, mais leurs compétences étaient exceptionnelles. Vous avec besoin d'un mécanicien de vélos du Tour de France? Pas de problème, allez parler au gars qui organise la logistique à la base. Vous pensez organiser un concert? Facile, allez au centre de gestion du virus Ebola et demandez l'infirmière d'Ottawa, qui est également violoniste concertiste. Vous avez un bateau à conduire des Caraïbes à l'Europe? Demandez au gars chargé de l'assainissement de l'eau. Lorsqu'il n'est pas en train de parcourir le monde en yacht ou de parcourir l'Afrique à moto, il peut entreprendre ce voyage pour vous, en solo. Incidents chimiques, biologiques, nucléaires? Gestion des traumatismes? Nous avons un médecin/infirmier néerlandais qui peut se charger de tout ça. Je me suis lavé de nouveau les mains à l'eau de javel et je me suis assis au milieu de ce groupe très talentueux pour un souper composé de poulet et de riz, le premier d'une série de trente. Ensuite, j'ai pris la première des trente douches froides et c'est seulement là que j'ai pu ramper sous ma moustiquaire pour aller dormir. Le lendemain était une journée de travail!

Le matin suivant, j'ai attrapé mon Malarone, un complexe multivitaminé et mon flacon plus qu'essentiel de Purell, puis je suis parti avec mes collègues pour le centre de gestion du virus Ebola afin d'assister à une séance d'information de transfert de l'équipe qui était de permanence au laboratoire mobile depuis le $1^{\mathrm{er}}$ décembre. Tous les quatre, nous avons sauté dans le Land Cruiser et le chauffeur a appelé la salle des radios "Kilo Base... Kilo Base. Ici Bravo-4. J'ai quatre Echo (expatriés) à bord. Direction le centre de gestion du virus Ebola. Terminé. » Les portes en acier de Kilo Base se sont ouvertes et nous nous sommes aventurés dehors, dans le demi-jour.

Le premier de mes voyages quotidiens a commencé par la traversée d'un beau paysage pastoral semi-rural peuplé uniquement par des chiens endormis et quelques voyageurs de la nuit fantomatiques qui transportaient avec précaution leurs affaires sur leur tête. Nous sommes ensuite passés par la petite ville de Kailahun qui est le centre commercial et bureaucratique du district servant de premier port d'escale pour les marchandises en provenance de Guinée. Dans l'aube naissante, j'ai pu voir des bâtiments à moitié détruits et des maisons brûlées laissés debout comme pour témoigner de la violence de la guerre civile terminée plus d'une décennie plus tôt en 2002. Le camion est passé devant des panneaux municipaux délavés de la fierté nationale proclamant la renaissance de la Sierra Leone avec des slogans appelant à la coopération et à la guérison. Nous sommes passés devant la mosquée, à côté de l'église évangélique et, alors que nous gravissions une autre colline, la route s'est divisée en deux en raison d'une tranchée formée par la saison des pluies terminée depuis un mois. La ville commençait à s'éveiller. J'ai vu des gens se brosser les dents à côté de la route, des mères cuisiner le petitdéjeuner sur un feu en plein air, et des nuées de petits enfants criant « Poomway » (personne blanche) en faisant de grands signes de la main avec l'espoir d'obtenir un signe de la main en retour. Le B-4 a fait une embardée et a grincé alors qu'il gravissait encore une colline, puis nous avons pris un virage à gauche et j'ai vu pour la première fois le centre de gestion du virus Ebola au loin se détacher littéralement de la jungle.

Lorsque nous avons quitté l'éclatant soleil africain pour entrer dans la structure semi-permanente assombrie des vestiaires, le rituel du nettoyage à l'eau de javel et de la prise de température s'est répété. Désorienté parmi la foule de gens, on m'a dit de demander des gants verts et des bottes en caoutchouc blanches, la tenue de combat contre le virus Ebola. Le commis m'a remis des vêtements qui ne m'allaient pas du tout mais j'étais trop embarrassé pour en demander d'autres et je suis sorti des vestiaires de façon inconvenante. J'ai boitillé le long de la zone neutre jonchée de pierres entre la zone à risque élevé (patients infectés par le virus Ebola) et la zone à risque faible où le laboratoire était situé, en portant une botte qui était trop grande et une botte qui était bien 
trop petite. Alors que je passais à côté de rangées d'énormes tentes en toiles drapées de pare-soleil ou prolongées par des toits en tôle ondulée, le personnel m'interpellait amicalement « Bee-ay-ee! » « Vous avez bien dormi? ». Je ne savais pas quoi répondre, je souriais donc d'un sourire étonné et me dirigeais vers les tentes de laboratoire atteintes de mildiou qui se trouvait tout au bout du centre de gestion du virus Ebola.

Lorsque je suis arrivé, j'ai ouvert les rabats de la première tente et je suis entré. J'ai vu quelques instruments familiers et un congélateur mais, je suis entré dans un monde totalement inconnu lorsque j'ai vu la tente d'isolement où nous ouvrions les échantillons du virus Ebola. Tout l'équipement était posé sur des tables en bois flache placées sur une bâche usée couverte d'une poussière rouge omniprésente. Cela ne ressemblait à aucun des laboratoires où j'avais travaillé auparavant.

M'abandonner à la Sierra Leone, c'était changer mon point de vue à de très nombreux niveaux. À Ottawa, j'avais consacré des heures à soigneusement élaborer des lignes directrices pour manipuler de façon sécuritaire les échantillons potentiels du virus Ebola, dans les conditions les plus strictes possible. À l'extérieur de mon nouveau laboratoire, à trois pieds de moi, se trouvaient trois seaux en polyéthylène blanc dans le gravier rouge du centre de gestion du virus Ebola et ceux-ci étaient pleins d'échantillons potentiels du virus Ebola. Alors que je m'efforçais à faire le rapprochement entre ces milieux complètement différents, je me suis presque évanoui lorsqu'un membre de l'équipe sur le départ a pulvérisé un couvercle d'eau de javel et a ouvert le conteneur pour compter le nombre d'échantillons à l'intérieur. J'ai sorti nerveusement mon flacon de Purell, j'ai reculé de trois pas et j'ai regardé mon collègue qui, à mon grand étonnement, était toujours vivant et me regardait fixement. II a demandé : « Bon, tu vas tester ces échantillons ou pas? ». J'ai saisi le protocole maintes fois consulté que j'avais soigneusement annoté pendant la formation au LNM et relu huit fois dans l'avion et j'ai pensé : «Par où commencer? ». J'ai nerveusement jeté un coup d'œil autour pour gagner du temps et j'ai vu un flacon pompe géant de Purell dans le laboratoire. J'ai appuyé cinq longues fois sur la pompe. C'était bon de frotter le produit sur mes mains et j'ai senti mon courage revenir. J'ai pris ma respiration et j'ai décidé que le mieux serait de commencer par l'étape 1 du protocole.

J'avais une excellente partenaire de laboratoire avec laquelle j'ai partagé les tâches de laboratoire, Qiu. C'est en quelque sorte un ponte, car elle a développé ZMapp, les anticorps monoclonaux qui constituent probablement le traitement le plus efficace contre le virus Ebola de la planète. Elle manipulait les échantillons coriaces, portant l'intégralité de l'équipement de protection individuelle et neutralisant les échantillons « chauds » avant de me les transmettre, mais j'étais quand même essentiel à l'opération. J'ai réussi à obtenir la connexion à Internet par satellite dans la matinée, puis j'ai raccordé le routeur aux machines à PCR et j'ai retiré les insectes, les lézards et les rats de la tente. Je lui ai rappelé que je n'étais pas complètement inutile. Mes autres tâches comprenaient la purification de l'ARN viral des échantillons neutralisés, puis l'exécution du test Ebola. Mais il faut être vraiment être clair sur un point : j'étais juste un gars qui avait un passeport, beaucoup de soutien et une expérience en laboratoire. La magie opérait grâce aux gens du laboratoire des pathogènes spéciaux du LNM qui ont élaboré les tests et qui ont travaillé dans les laboratoires mobiles pendant la majeure partie des éclosions de la maladie à virus Ebola en Afrique.

Au cours du mois suivant, dans deux tentes au milieu de la jungle et équipés d'un générateur diesel, nous avons effectué des centaines de tests Ebola sans la moindre faille. Après avoir travaillé dans un laboratoire diagnostique comme le LNM, il était étonnant de voir ce laboratoire de terrain obtenir des résultats aussi robustes et fiables que ceux qui seraient obtenus dans les laboratoires conventionnels. Dès notre première journée, nous avons effectué des tests pour Ebola ainsi que pour la malaria et la fièvre de Lassa. Cette journée-là, nous n'avons pas trouvé le virus Ebola, seulement la malaria. Une fois le travail de laboratoire complété et l'équipement décontaminé, j'ai rapporté les résultats à l'équipe médicale du centre de gestion du virus Ebola, changé mes vêtements serrés, désinfecté mes mains encore une fois puis monté à bord de la Land Cruiser pour retourner à Kilo Base.

Après avoir mangé les mêmes repas, effectué les mêmes trajets, porté les mêmes vêtements serrés, effectué les mêmes tests de laboratoire pendant 30 jours consécutifs, l'aventure prenait fin. Qui avait transféré dans un autre laboratoire de terrain et je me retrouvais seul au centre de gestion du virus Ebola. Au coucher du soleil, j'ai regardé la fumée s'échapper des tentes vacantes qui brûlaient dans la zone à haut risque. En tant que dernier homme sur place au sein du centre de gestion du virus Ebola, qui était dépourvu de cas Ebola, j'ai regardé autour de moi et su que mon travail était terminé. J'ai rangé le matériel du laboratoire dans la camionnette, nettoyé mes mains avec du Purell, monté à bord du B-4 puis écouté pour une dernière fois le conducteur faire l'appel radio : "Kilo Base, Kilo Base. J'ai un Echo (expatrié) à bord. Nous sommes en route". Je m'en retournais à la maison.

Mes valises étaient chargées à l'arrière du B-4 alors que j'attendais à Kilo Base. J'ai entendu le bruit d'un hélicoptère qui devenait de plus en plus fort, l'indice que je devais courir vers le B-4 en route vers le stade de soccer où je rencontrerais l'hélicoptère des Nations Unies qui m'amènerait à Freetown. Au moment où l'hélicoptère prend son envol et en saluant les enfants dispersés comme des feuilles dans le vent, je débute ma réflexion sur l'expérience que je viens de vivre. Je pensais aux relations qui se sont créées avec le personnel sur place et leurs efforts pour m'enseigner la langue locale Mende. Je m'émerveillais de la synergie entre le laboratoire mobile du LNM et MSF. La réputation de MSF nous a permis de travailler de façon sécuritaire et efficace en Sierra Leone. L'organisation était très bien organisée et elle a apporté une immense contribution à la lutte contre l'épidémie Ebola dans l'ouest de l'Afrique de par son assistance dès les premiers moments. Cependant, MSF n'avait pas de laboratoire pour effectuer des tests Ebola sur des patients ou, lorsque les patients survivaient, pour déterminer si les patients pouvaient obtenir leur congé et retourner auprès de leur famille. Le laboratoire mobile a permis au centre de gestion du virus Ebola d'opérer. Bien que je n'aie pas vu de cas Ebola durant mes 30 jours passés en Sierra Leone, les 450 corps étendus dans le cimetière à côté du centre de gestion du virus Ebola témoignaient silencieusement de la récente présence d'Ebola. Durant les six mois au cours desquels le laboratoire de terrain fut en opération, plus de 2700 tests furent effectués sur 1200 admissions. Au total, vingt-et-un employés remarquables de l'Agence de la santé du Canada ont sillonné le Sierra Leone afin de doter les laboratoires mobiles situés à Kailahun et Magburaka, chacun apportant une énorme contribution au nom du Canada. 